\title{
Neutrino masses and lepton flavor violation in the 3-3-1 model with right-handed neutrinos
}

\author{
P. V. Dong* \\ Theory Group, KEK, 1-1 Oho, Tsukuba, 305-0801, Japan \\ H. N. Long甲 \\ Institute of Physics, VAST, P.O. Box 429, Bo Ho, Hanoi 10000, Vietnam
}

(Dated: August 31, 2021)

\begin{abstract}
We show that in the framework of the 3-3-1 model with right-handed neutrinos, small neutrino masses and large lepton flavor violating processes such as $\mu \rightarrow 3 e$ and $\mu \rightarrow e \gamma$ can be obtained by just introducing an additional Higgs sextet. In the limit of vanishing of the Yukawa interaction among Higgs and lepton triplets $\left(h^{\nu}=0\right)$, the decay $\mu \rightarrow 3 e$ strongly depends on the neutrino mass patterns, but the $\mu \rightarrow e \gamma$ almost does not. The neutrino masses are not constrained by such processes in the cases of $h^{\nu} \neq 0$.

PACS numbers: 14.60.Pq, 13.35.Bv, 12.60.Fr, 12.60.Cn
\end{abstract}

\section{INTRODUCTION}

The recent experimental results confirm that neutrinos have tiny masses and oscillate [1], this implies that the standard model (SM) must be extended. Among the beyond-SM extensions, the models based on the $\mathrm{SU}(3)_{C} \otimes \mathrm{SU}(3)_{L} \otimes \mathrm{U}(1)_{X}$ (3-3-1) gauge group [2, 3] have some intriguing features: First, they can give partial explanation of the generation number problem. Second, the third quark generation has to be different from the first two, so this leads to the possible explanation of why top quark is uncharacteristically heavy. An additional motivation to study this kind of the models is that they can also predict the electric charge quantization [4].

Such 3-3-1 models have been studied extensively over the last decade. In one of them the three lepton triplets are of the form $\left(\nu, l, \nu^{c}\right)_{L}$, where the right-handed $(\mathrm{RH})$ neutrinos $\nu_{R}^{c}=\left(\nu^{c}\right)_{L}$ are included into the third components of triplets. Traditionally, this model works with the three Higgs triplets and named the 3-3-1 model with RH neutrinos [3]. At the tree level the neutrino spectrum contains three Dirac fields with one massless and two degenerate in mass $\sim h^{\nu} v$, where the $v$ vacuum expectation value (VEV) is related to the electroweak scale, and the Majorana fields $\nu_{L}$ and $\nu_{R}$ are massless. This spectrum is not realistic under the data because there is only one squared-mass splitting. Since the observed neutrino masses are so small, the Dirac mass is unnatural, and one must understand what physics gives $h^{\nu} v \ll h^{l} v$ - the mass of charged leptons. The neutrino masses are thus a great question addressed to this kind of the models.

Alternatively, the neutrino oscillation shows clearly that the lepton flavors are not conserved in nature. If we accommodate this feature simply by an introduction

\footnotetext{
*Electronic address: pvdong@iop.vast.ac.vn On leave of absent from Institute of Physics, VAST, Vietnam

${ }^{\dagger}$ Electronic address: hnlong@iop.vast.ac.vn
}

of neutrino masses in the SM, other lepton flavor violating (LFV) processes such as $\mu \rightarrow e \gamma$ would still have so small a rate (branching ratio $\leq 10^{-40}$ ) that there is no hope for their detection in the foreseeable future [ $[$, 6 ]. This is also the case of the model under consideration. The LFV processes such as $\mu \rightarrow e \gamma$ and $\mu \rightarrow 3 e$ found are very suppressed too. In this work, we are particularly interested in the possibility of generating small neutrino masses so that the LFV processes are less damped. We stress that this might be achieved by introducing just an additional Higgs sextet. This sextet now becomes a nice element because the neutrino mass is the result of a type II seesaw [7] and the LFV processes in a case are mediated only by the doubly-charged scalar [8]. The sterile neutrinos mix small with the ordinary ones and have masses in the 3-3-1 symmetry breaking scale.

This work is organized as follows. In Sec. III we present the model. Section 【II introduces the Higgs sextet and give a realistic mass spectrum for the neutrinos. Section IV is devoted to the LFV processes $\mu \rightarrow 3 e$ and $\mu \rightarrow$ $e \gamma$. Phenomenological relationship among the neutrino spectra and LFV decays is obtained in the end of this section. We make conclusions in Section $\nabla$.

\section{A REVIEW OF THE MODEL}

The model under consideration is of the 3-3-1 model with RH neutrinos [3]. The particle content which is anomaly free is given as follows $\psi_{a L}=\left(\nu_{a L}, l_{a L}, \nu_{a R}^{c}\right)^{T} \sim$ $(3,-1 / 3) \quad(a=1,2,3), \quad l_{a R} \sim(1,-1), \quad Q_{3 L}=$ $\left(u_{3 L}, d_{3 L}, U_{L}\right)^{T} \sim(3,1 / 3), Q_{\alpha L}=\left(d_{\alpha L},-u_{\alpha L}, D_{\alpha L}\right)^{T} \sim$ $\left(3^{*}, 0\right)(\alpha=1,2), u_{a R} \sim(1,2 / 3), d_{a R} \sim(1,-1 / 3), U_{R} \sim$ $(1,2 / 3), D_{\alpha R} \sim(1,-1 / 3)$. The values in the parentheses denote quantum numbers based on the $\left(\mathrm{SU}(3)_{L}, \mathrm{U}(1)_{X}\right)$ symmetry. The electric charge operator takes a form $Q=T_{3}-\frac{1}{\sqrt{3}} T_{8}+X$, where $T_{i}(i=1,2, \ldots, 8)$ and $X$ stand for $\mathrm{SU}(3)_{L}$ and $\mathrm{U}(1)_{X}$ charges, respectively. The electric charges of exotic quarks are the same as of the 
ordinary ones: $q_{U}=\frac{2}{3}$ for $U$ and $q_{D}=-\frac{1}{3}$ for $D_{\alpha}$.

The electroweak symmetry breaking in this model is through two stages, $\mathrm{SU}(3)_{L} \otimes \mathrm{U}(1)_{X} \rightarrow \mathrm{SU}(2)_{L} \otimes$ $\mathrm{U}(1)_{Y} \rightarrow \mathrm{U}(1)_{Q}$, achieved by three Higgs triplets: $\chi=\left(\chi_{1}^{0}, \chi_{2}^{-}, \chi_{3}^{0}\right)^{T} \sim(3,-1 / 3), \eta=\left(\eta_{1}^{0}, \eta_{2}^{-}, \eta_{3}^{0}\right)^{T} \sim$ $(3,-1 / 3), \quad \rho=\left(\rho_{1}^{+}, \rho_{2}^{0}, \rho_{3}^{+}\right)^{T} \sim(3,2 / 3)$, with the VEVs corresponding to $\langle\chi\rangle=\left(u^{\prime} / \sqrt{2}, 0, w / \sqrt{2}\right)^{T},\langle\eta\rangle=$ $\left(u / \sqrt{2}, 0, w^{\prime} / \sqrt{2}\right)^{T},\langle\rho\rangle=(0, v / \sqrt{2}, 0)^{T}$. The most general Yukawa Lagrangian responsible for fermion masses is separated into two parts:

$$
\begin{aligned}
\mathcal{L}_{\mathrm{LNC}}= & h^{U} \bar{Q}_{3 L} \chi U_{R}+h_{\alpha \beta}^{D} \bar{Q}_{\alpha L} \chi^{*} D_{\beta R}+h_{a}^{u} \bar{Q}_{3 L} \eta u_{a R} \\
& +h_{\alpha a}^{d} \bar{Q}_{\alpha L} \eta^{*} d_{a R}+h_{a}^{d} \bar{Q}_{3 L} \rho d_{a R}+h_{\alpha a}^{u} \bar{Q}_{\alpha L} \rho^{*} u_{a R} \\
& +h_{a b}^{l} \bar{\psi}_{a L} \rho l_{b R}+h_{a b}^{\nu} \bar{\psi}_{a L}^{c} \psi_{b L} \rho+\text { H.c. } \\
\mathcal{L}_{\mathrm{LNV}}= & s_{a}^{u} \bar{Q}_{3 L} \chi u_{a R}+s_{\alpha a}^{d} \bar{Q}_{\alpha L} \chi^{*} d_{a R}+s^{U} \bar{Q}_{3 L} \eta U_{R} \\
& +s_{\alpha \beta}^{D} \bar{Q}_{\alpha L} \eta^{*} D_{\beta R}+s_{\alpha}^{D} \bar{Q}_{3 L} \rho D_{\alpha R}+s_{\alpha}^{U} \bar{Q}_{\alpha L} \rho^{*} U_{R} \\
& + \text { H.c. },
\end{aligned}
$$

where the subscripts LNC and LNV respectively indicate to the lepton number conserving and violating ones as shown below.

The Yukawa couplings of (11) possess an extra global symmetry which is not broken by $u, v, w$ but by $u^{\prime}, w^{\prime}$. From these couplings, one can find the following lepton symmetry: $L\left(\nu_{a L}, l_{a L, R}, \nu_{a R}\right)=1$, $L\left(\chi_{1}^{0 *}, \chi_{2}^{+}, \rho_{3}^{+}, \eta_{3}^{0}, U_{L, R}, D_{\alpha L, R}^{*}\right)=-2$, and $L=0$ for other fields. Here the $L$ is broken by $u^{\prime}$ and $w^{\prime}$ due to $L\left(\chi_{1}^{0}, \eta_{3}^{0}\right) \neq 0$. It is interesting that the exotic quarks also carry the lepton number, so called leptoquarks. This $L$ obviously does not commute with the gauge symmetry, one can construct a new conserved charge $\mathcal{L}$ through $L$ by making a linear combination $L=x T_{3}+y T_{8}+\mathcal{L} I$. Applying $L$ on a lepton triplet, the coefficients will be determined $L=\frac{4}{\sqrt{3}} T_{8}+\mathcal{L} I$, where $\mathcal{L}(\chi)=4 / 3, \mathcal{L}\left(\eta, \rho, Q_{3 L}, Q_{\alpha L}^{*}\right)=-2 / 3, \mathcal{L}\left(u_{a R}, d_{a R}\right)=0$, $\mathcal{L}\left(U_{R}, D_{\alpha R}^{*}\right)=-2, \mathcal{L}\left(\psi_{a L}\right)=1 / 3$, and $\mathcal{L}\left(l_{a R}\right)=1$. Another useful conserved charge $\mathcal{B}$ exactly not broken by any VEV is usual baryon number: $B=\mathcal{B} I$, where $\mathcal{B}=1 / 3$ for all the quark multiplets and $\mathcal{B}=0$ for Higgs and lepton ones. It is noteworthy that although $\chi$ and $\eta$ have the same quantum numbers, they are discriminative due to difference in $\mathcal{L}$-charge.

The interactions (2) violate $\mathcal{L}$ with \pm 2 units. In addition they imply mixing among the exotic quarks and ordinary quarks of the same charge: $\left(u_{1}, u_{2}, u_{3}, U\right)$ and $\left(d_{1}, d_{2}, d_{3}, D_{1}, D_{2}\right)$, this would lead to the flavor-changing neutral-current processes [9]. By those reasons, it should be noted that the Yukawa couplings (2) must be respectively much smaller than the first ones (1), $s \ll h$. Also, the lepton number breaking VEVs are respectively much smaller than the usual ones, $u^{\prime} \ll u$ and $w^{\prime} \ll w$. In this case all the quarks get mass at the tree level. The Lagrangian (2) has often been excluded commonly by the adoption of an appropriate discrete symmetry [9, 10], but there is no reason within the 3-3-1 models why such Lagrangian should not be present.

The mass matrix for the charged leptons is obtained by $M_{l}=-\frac{1}{\sqrt{2}} v h^{l}$, which is the same as in the ordinary version [3]. Hereafter, we will assume that $h^{l}$ is flavor diagonal, thus $l_{a}$ are mass eigenstates with respective to mass eigenvalues $m_{a}=-\frac{1}{\sqrt{2}} v h_{a a}^{l}$.

The Lagrangian for the tree-level neutrino masses is obtained as

$$
\mathcal{L}_{\text {mass }}^{\nu}=-\left(M_{D}\right)_{a b} \bar{\nu}_{a R} \nu_{b L}+\text { H.c. }, \quad M_{D}=-\sqrt{2} v h^{\nu},
$$

where $h_{a b}^{\nu}$ is antisymmetry in $a$ and $b$ due to Fermi statistics. The tree-level spectrum therefore consists of only Dirac neutrinos with one particle massless and two others degenerate in mass: $0,-m, m$. This spectrum is unrealistic, but it could be severely changed by the quantum corrections. In this case both the Dirac and Majorana mass types get the possible corrections.

If such a tree-level spectrum dominates resulting masses after the corrections, the model provides a possible explanation of the large splitting $\Delta m_{\mathrm{atm}}^{2} \gg \Delta m_{\mathrm{sol}}^{2}$. But we then must need a fine-tuning $m \sim\left(\Delta m_{\text {atm }}^{2}\right)^{1 / 2} \sim$ $5 \times 10^{-2} \mathrm{eV}$, thus $h^{\nu} \sim 10^{-13}[1]$. The coupling $h^{\nu}$ is so small and therefore this fine-tuning is unnatural. Exactly, $h^{\nu}$ enter the radiative corrections for the neutrino Majorana masses, these masses would get so small values not large enough to split the degenerate masses into a realistic spectrum (the largest splitting in squared-mass is still much smaller than $\Delta m_{\mathrm{sol}}^{2} \sim 8 \times 10^{-5} \mathrm{eV}^{2}[1]$ ). In this case, the Dirac masses get trivially corrections [11]. Those conclusions are in contradiction with the previous one given in 12].

We could introduce an appropriate discrete symmetry to suppress the tree-level neutrino masses so that the neutrino spectrum is entirely induced by either radiative corrections [10] or effective operators [13]. In such cases, if one takes an examination on the LFV processes such as $\mu \rightarrow e \gamma$ and $\mu \rightarrow 3 e$, the branching ratios are found to be very suppressed (cf. [5]). If any detection of such processes is positive, the models are thus no longer favored. In the following we will search for the possibility of large LFV processes while still keeps naturally-small neutrino masses.

\section{HIGGS SEXTET AND NEUTRINO MASS}

The neutrino Dirac masses by their naturalness will be treated as large as of the usual charged ones resulting from the standard symmetry breaking. We shall solve the problems as mentioned by introducing just an additional Higgs sextet into the model. The sextet has been considered formerly in 9, 14 in order to accommodate for the neutrino masses, but the correct formulation of neutrinomass matrix has not been given. The physics associated with this sextet has not yet been explored. New interesting phenomenologies concerning the neutrino mass spectra and LFV processes are to be studied in this work.

Decomposing $\bar{\psi}_{a L}^{c} \psi_{b L} \sim\left(3^{*}+6,-2 / 3\right)$, the values $\left(3^{*},-2 / 3\right)$ are just charges coupled to $\rho^{*}$. The remaining 
$(6,-2 / 3)$ has been leaved before and now assigned to a Higgs sextet. This sextet and its VEVs are, respectively, defined by

$$
S=\left(\begin{array}{ccc}
S_{11}^{0} & S_{12}^{-} & S_{13}^{0} \\
S_{12}^{-} & S_{22}^{-} & S_{23}^{-} \\
S_{13}^{0} & S_{23}^{-} & S_{33}^{0}
\end{array}\right), \quad\langle S\rangle=\frac{1}{\sqrt{2}}\left(\begin{array}{ccc}
\kappa & 0 & \vartheta \\
0 & 0 & 0 \\
\vartheta & 0 & \Lambda
\end{array}\right)
$$

It has the following Yukawa couplings:

$$
\mathcal{L}_{S}=f_{a b}^{\nu}\left(\bar{\psi}_{a L}^{c}\right)_{m}\left(\psi_{b L}\right)_{n}\left(S^{*}\right)_{m n}+\text { H.c. }
$$

where $f_{a b}^{\nu}$ is symmetry in $a$ and $b$. The $S_{22}^{--}$is the unique particle in the model carrying the exotic charge "-_", it does not mix with other particles and hence becomes a physical scalar with mass $M$. One can check the lepton charge $\mathcal{L}(S)=\frac{2}{3}$, thus $L\left(S_{33}^{0}\right)=-2$ and $L\left(S_{11}^{0}, S_{12}^{-}, S_{22}^{--}\right)=2$ (other components have vanishing $L)$. The charged $S_{12}^{-}, S_{22}^{--}$are bilepton particles, and the neutral components $S_{11}^{0}, S_{33}^{0}$ with respective to VEVs $\kappa$, $\Lambda$ beak the lepton number responsible for the left- and right-handed neutrino Majorana masses, respectively.

Decomposing the Higgs multiplets into the SM ones, we get four doublets $\left(\chi_{1}^{0}, \chi_{2}^{-}\right)^{T},\left(\eta_{1}^{0}, \eta_{2}^{-}\right)^{T},\left(\rho_{1}^{+}, \rho_{2}^{0}\right)^{T}$ and $\left(S_{13}^{0}, S_{23}^{-}\right)^{T}$, a triplet $\left(S_{11}^{0}, S_{12}^{-}, S_{22}^{--}\right)$, and four singlets $\chi_{3}^{0}, \eta_{3}^{0}, \rho_{3}^{+}$and $S_{33}^{0}$. The VEVs of the singlet components $w$ and $\Lambda$ give mass for exotic quarks and RH Majorana neutrinos as well as the new gauge bosons. The VEVs of doublets and triplet give mass for all the ordinary fermions and gauge bosons. To keep a consistency with the effective theory, including the conditions as given previously, it is safe to impose the constraints: $w^{\prime} \ll w$ and $u^{\prime}, \kappa \ll u, v, \vartheta \ll w, \Lambda$. In this effective limit, the mass of $W$ boson and the $\rho$-parameter are evaluated by $M_{W}^{2} \simeq \frac{g^{2}}{4}\left(u^{2}+v^{2}+2 \vartheta^{2}\right), \rho=\frac{M_{W}^{2}}{c_{W}^{2} M_{Z}^{2}} \simeq 1-\frac{2 \kappa^{2}}{u^{2}+v^{2}+2 \vartheta^{2}}$. We therefore identify $u^{2}+v^{2}+2 \vartheta^{2}=v_{\text {weak }}^{2} \simeq(246 \mathrm{GeV})^{2}$, and then obtain the limit $|\kappa|<2.46 \mathrm{GeV}$ with the help of the data $\rho>0.9998[1]$.

With the aid of (11) and (5), the Lagrangian (3) is rewritten in the form:

$$
\mathcal{L}_{\text {mass }}^{\nu}=-\frac{1}{2}\left(\bar{\nu}_{L}^{c}, \bar{\nu}_{R}\right) M_{\nu}\left(\begin{array}{c}
\nu_{L} \\
\nu_{R}^{c}
\end{array}\right)+\text { H.c. },
$$

where the mass matrix for the neutrinos is obtained as follows

$$
M_{\nu}=-\sqrt{2}\left(\begin{array}{cc}
\kappa f^{\nu} & \left(v h^{\nu}+\vartheta f^{\nu}\right)^{T} \\
v h^{\nu}+\vartheta f^{\nu} & \Lambda f^{\nu}
\end{array}\right) .
$$

Let us remind the reader that the first term of the Dirac mass matrix, $v h^{\nu}$, was excluded in the latter one of [14]. Because $\kappa \ll v, \vartheta \ll \Lambda$, the active neutrinos $\sim \nu_{L}$ gain mass via a type II seesaw:

$$
M_{1} \simeq-\sqrt{2}\left\{\left(\kappa-\frac{\vartheta^{2}}{\Lambda}\right) f^{\nu}-\frac{v^{2}}{\Lambda} h^{\nu}\left(f^{\nu}\right)^{-1} h^{\nu}\right\} .
$$

It turns out that the neutrino masses in this model are naturally small because of suppression of a large $\Lambda$ scale and a small $\kappa$ constrained from the $\rho$-parameter. The sterile neutrinos $\sim \nu_{R}$ have large masses in the $\Lambda$ scale: $M_{2} \simeq-\sqrt{2} \Lambda f^{\nu}$. Because $f^{\nu}$ and $h^{\nu}$ are, respectively, symmetry and antisymmetry, the Dirac $v h^{\nu}+\vartheta f^{\nu}$ in (7) is an arbitrary complex matrix. This means that the seesaw mechanism as given in the model is quite general. As shown below the coupling $f^{\nu}$ is more constrained by the LFV processes but $h^{\nu}$ does not. This actually allows us to recover appreciate neutrino-mass spectra by $h^{\nu}$ while keep the LFV branching ratios in the present bounds.

Let us give a numerical estimation. Putting $u \sim v \sim$ $\vartheta \sim 100 \mathrm{GeV}$ from the $W$ mass, $\kappa \Lambda \sim \vartheta^{2}, f^{\nu} \sim h^{\nu}$, and $M_{1} \sim 1 \mathrm{eV}$, the sterile masses are proportional to $M_{2} \sim$ $\left(h^{\nu}\right)^{2} \frac{v^{2}}{M_{1}} \sim\left(h^{\nu}\right)^{2} \times 10^{13} \mathrm{GeV}$. We see that the seesaw scale $M_{2} \sim 1 \mathrm{TeV}$ if $h^{\nu}$ is in order of the electron Yukawa coupling $h^{\nu} \sim 10^{-5}$, and $M_{2} \sim 10^{8} \mathrm{GeV}$ if $h^{\nu}$ rises to the muon or tauon Yukawa coupling. For simplicity, in the following we will take the last or higher value of $M_{2}$ into account, i.e. the contribution to $\mu \rightarrow e \gamma$ shall entirely come from the doubly-charged scalar and the values $f^{\nu} \sim$ $10^{-3}-10^{-2}$ follow.

\section{LEPTON FLAVOR VIOLATING PROCESSES}

In this model, the process $\mu \rightarrow 3 e$ is given at a tree level diagram as mediated by the doubly-changed scalar. The branching ratio is obtained by

$$
\operatorname{Br}(\mu \rightarrow 3 e) \simeq \frac{\Gamma(\mu \rightarrow 3 e)}{\Gamma\left(\mu \rightarrow e \tilde{\nu}_{e} \nu_{\mu}\right)} \simeq \frac{1}{4 G_{F}^{2}} \frac{\left|f_{11}^{\nu \dagger} f_{12}^{\nu}\right|^{2}}{M^{4}}
$$

where $G_{F}=1.16637 \times 10^{-5} \mathrm{GeV}^{-2}$ is the Fermi constant [1]. Taking $M \sim(200-1000) \mathrm{GeV}$ and $\left|f_{11}^{\nu \dagger} f_{12}^{\nu}\right|=10^{-6}$ as mentioned above, we get $\operatorname{Br}(\mu \rightarrow 3 e) \sim 10^{-12}-$ $1.8 \times 10^{-15}$ which coincides with the current experimental bound: $\operatorname{Br}(\mu \rightarrow 3 e) \leq 10^{-12}[1]$.

The process $\mu \rightarrow e \gamma$ is given at one-loop level diagrams as mediated by the charged leptons $(e, \mu, \tau)$ and doubly-charged scalar. There are two kinds of the diagrams among them corresponding to photon emission from internal scalar and fermion lines which yield relevant amplitudes. Other diagrams with photon emission from external charged lines contribute only to the $\bar{e} \gamma^{\lambda} \mu \epsilon_{\lambda}^{*}$ amplitude which vanishes because of current conservation. The branching ratio is given by

$$
\operatorname{Br}(\mu \rightarrow e \gamma) \simeq \frac{\alpha}{3 \pi G_{F}^{2}} \frac{\left|\left(f^{\nu \dagger} f^{\nu}\right)_{12}\right|^{2}}{M^{4}}
$$

where $\alpha=e^{2} / 4 \pi=1 / 128$ [1]. Taking $\left|\left(f^{\nu \dagger} f^{\nu}\right)_{12}\right|=10^{-4}$ and $M=(200-1000) \mathrm{GeV}$ as mentioned, we obtain $\operatorname{Br}(\mu \rightarrow e \gamma) \sim 3.8 \times 10^{-11}-6 \times 10^{-14}$ which is comparable to the bound of current experiments: $\operatorname{Br}(\mu \rightarrow e \gamma) \leq$ $1.2 \times 10^{-11}[1]$.

At this one-loop level, there are other sources contributing to $\mu \rightarrow e \gamma$. The first one is mediated by charged 
gauge bosons - the SM $W^{ \pm}$and bilepton $Y^{ \pm}$because the neutrinos mix, but these contributions are so small and thus safely neglected [5]. The second comes from physical singly-changed scalars similarly to the case of the doubly-charged scalar, but now the internal fermion lines are neutrinos (in more details, see [6]). The contribution is quite smaller than those given in (10) and can neglect if $M_{1}$ is in $\mathrm{eV}, M_{2}$ of order $\mathcal{O}\left(10^{8}-10^{16}\right) \mathrm{GeV}$, and the singly-charge scalar masses are the same order of doublycharged one: $\mathcal{O}(100-1000) \mathrm{GeV}$. In another scenario the seesaw scale $M_{2}$ is in $\mathrm{TeV}$, that contribution is comparable to (10). Without loss of generality we do not consider the case in this work because we are interested only in the LFV size of the decay.

The relationship among neutrino mass spectra and LFV rates can be divided into: Case $1 . h^{\nu}=0$ : The mass matrix of neutrinos (8) is rewritten as $M_{1}=$ $-\sqrt{2}\left(\kappa-\frac{\vartheta^{2}}{\Lambda}\right) f^{\nu}$. Thus the neutrino masses and LFV muon decays depend only on $f^{\nu}$ which is similar to Higgs triplet model [15, 16], but in our case neutrinos gain generic masses from a type II seesaw. Various neutrino mass patterns could be distinguished by measuring LFV processes as explored in [16]. The decay $\mu \rightarrow 3 e$ is significantly enhanced in the case of degenerate or invertedhierarchical masses compared with that of the normal hierarchy, whereas the rate of $\mu \rightarrow e \gamma$ is almost insensitive to these mass patterns. Case 2. $h^{\nu} \neq 0$ : Neutrino mass matrix takes the general form (8) depending on $h^{\nu}$ as well. We recall that most of $f^{\nu}$ are constrained by several LFV processes, but $h^{\nu}$ does not. The neutrino masses are not constrained by such processes. Thus there is no relationship among neutrino mass spectra and LFV rates, in comparison to the first case.

\section{CONCLUSIONS}

Origin 3-3-1 model with RH neutrinos could not generate consistent neutrino masses, simultaneously gives large LFV muon decays, which is the same as in simple extensions of the SM [5]. By introducing of the Higgs sextet, the neutrino masses are naturally small induced via a type II seesaw mechanism. The seesaw scale is one of the 3-3-1 symmetry breaking scales, it is signified in $\mathrm{TeV}$ order if the neutrino Dirac masses are around electron mass. But, it reaches $10^{8} \mathrm{GeV}$ whether the Dirac masses rise to muon or tauon mass. The neutrino mass matrix is given in the correct form revising that in [14].

LFV decays $\mu \rightarrow 3 e$ and $\mu \rightarrow e \gamma$ are mediated only by the doubly-charged Higgs sextet at high seesaw scales $\left(10^{8}-10^{16}\right) \mathrm{GeV}$, but in $\mathrm{TeV}$ scale the singly-charged scalars also contributing to $\mu \rightarrow e \gamma$. These decay rates are large and comparable to the current experimental bounds. In limit $h^{\nu}=0$, the neutrino mass and LFV happen similarly to the Higgs triplet model. On other cases of $h^{\nu} \neq 0$, the neutrino masses are almost not constrained by such lepton flavor violating processes.

\section{Acknowledgments}

P.V.D. is grateful to Nishina Fellowship Foundation for financial support. He would like to thank Prof. Y. Okada and Members of Theory Group at KEK for support and comments, and Prof. C.S. Lim at Kobe University for discussions. This work was also supported by National Council for Natural Sciences of Vietnam.
[1] For reviews, see: W. -M. Yao et. al. (Particle Data Group), J. Phys. G: Nucl. Part. Phys. 33, 1 (2006), and references therein.

[2] F. Pisano and V. Pleitez, Phys. Rev. D 46, 410 (1992); P. H. Frampton, Phys. Rev. Lett. 69, 2889 (1992); R. Foot, O. F. Hernandez, F. Pisano and V. Pleitez, Phys. Rev. D 47, 4158 (1993).

[3] M. Singer, J. W. F. Valle and J. Schechter, Phys. Rev. D 22, 738 (1980); R. Foot, H. N. Long and Tuan A. Tran, Phys. Rev. D 50, 34 (R) (1994); J. C. Montero, F. Pisano and V. Pleitez, Phys. Rev. D 47, 2918 (1993); H. N. Long, Phys. Rev. D 54, 4691 (1996); Phys. Rev. 53, 437 (1996).

[4] F. Pisano, Mod. Phys. Lett A 11, 2639 (1996); A. Doff and F. Pisano, Mod. Phys. Lett A 14, 1133 (1999); C. A. de S. Pires and O. P. Ravinez, Phys. Rev. D 58, 035008 (1998); C. A. de S. Pires, Phys. Rev. D 60, 075013 (1999); P. V. Dong and H. N. Long, Int. J. Mod. Phys. A 21, 6677 (2006).

[5] T. P. Cheng and L. F. Li, Phys. Rev. Lett. 45, 1908 (1980).

[6] B. He, T. P. Cheng and L. F. Li, Phys. Lett. B 553, 277 (2003).
[7] R. N. Mohapatra and G. Senjanović, Phys. Rev. D 23, 165 (1981); G. Lazarides, Q. Shafi and C. Wetterich, Nucl. Phys. B 181, 287 (1981); J. Schechter and J. W. F. Valle, Phys. Rev. D 25, 774 (1982).

[8] For a review, see: Y. Kuno and Y. Okada, Rev. Mod. Phys. 73, 151 (2001).

[9] J. C. Montero et. al. in Ref. 3].

[10] D. A. Gutierrez, W. A. Ponce and L. A. Sanchez, Int. J. Mod. Phys. A 21, 2217 (2006).

[11] P. V. Dong, H. N. Long and D. V. Soa, Phys. Rev. D 75, 073006 (2007).

[12] D. Chang and H. N. Long, Phys. Rev. D 73, 053006 (2006).

[13] A. G. Dias, C. A. de S. Pires and P. S. Rodrigues da Silva, Phys. Lett. B 628, 85 (2005).

[14] J. W. F. Valle and M. Singer, Phys. Rev. D 28, 540 (1983); N. A. Ky and N. T. H. Van, Phys. Rev. D 72, 115017 (2005).

[15] E. Ma, M. Raidal and U. Sarkar, Nucl. Phys. B 615, 313 (2001).

[16] E. J. Chun, K. Y. Lee and S. C. Park, Phys. Lett. B 566, 142 (2003); M. Kakizaki, Y. Ogura and F. Shima, Phys. Lett. B 566, 210 (2003). 